Original article

\title{
Loads on small muscle groups as a risk of hypertensive conditions
}

\author{
Olga G. Kourova, Yuri I. Koryukalov, Tatyana V. Popova \\ South Ural State University, Chelyabinsk, Russia
}

Received 5 March 2017, Revised 12 June 2017, Accepted 8 July 2017

(C) 2017, Kourova O.G., Koryukalov Y.I., Popova T.V.

(C) 2017, Russian Open Medical Journal

Abstract: Background - Hypertension is a widespread condition nowadays. Changes in physical activity patterns of the population, namely, sedentary lifestyle and increased loads on small muscle groups, are the key factors behind the development of hypertension. Although science has amassed sufficient amounts of facts about a hypertensive effect of local loads, the very mechanisms underlying adaptive reactions of the circulatory system have not received comprehensive study.

Material and Methods - We studied adaptive reactions to local muscle work in 108 adult subjects groups aged between 18 and 20,30 and 35, and 60 and 74 respectively by means of a Mosso's ergograph until the onset of fatigue with all the three age groups receiving medium loads. We have analyzed their work performance, including static and dynamic stamina. We took blood pressure measurements, electrocardiograms (ECGs) and electroencephalograms (EEGs) before and after the test.

Results - We discovered increased heartbeat rates, systolic blood pressure, and diastolic blood pressure in all of the subjects, as they were doing local load tests, while their ECGs showed shortened electric diastole time, which was indicative of heart functional tension, especially in the subjects aged between 18 and, and 60 and 74 . Adverse heart reactions were more pronounced while the subjects were doing static tests rather than dynamic tests, and their EEGs showed increased slow-wave activity within alpha- and theta-ranges, with regularly recurrent alpha wave synchronizations.

Conclusion - Our research shows that central mechanisms underlie hypertensive reactions of the cardiovascular system to local loads with the participation of metabolic receptors of muscles. We have also justified the necessity of preventive campaigns against hypertensions in individuals receiving increased amounts of local muscle work in the motor mode.

Keywords: hypertension, local loads, arterial blood pressure, electroencephalography, fatigue, prophylaxis.

Cite as Kourova OG, Koryukalov YI, Popova TV. Loads on small muscle groups as a risk of hypertensive conditions. Russian Open Medical Journal 2017; 6: e0407.

Correspondence to Tatyana V. Popova. Address: p.o. box 12385, Chelyabinsk, 454080, Russia. E-mail: tvpopova@susu.ru.

\section{Introduction}

Hypertension is a widespread condition that affects people of all ages. Physical activity may be a powerful preventive factor that enhances functional capabilities of physiological body systems [1, 2]. The researchers place an emphasis on muscular activity as a factor that provides the prophylaxis of early ageing of the body.

Insufficient physical activity, characteristic of most of age groups of population of developed countries is detrimental to adaptive capabilities of the body, especially those of the cardiovascular system. The cardiovascular system that provides adaptation to physical loads [3] is the weakest link in individuals who are prone to sedentary lifestyle.

Depending on a muscular volume that takes to do this or that kind of work, human physical loads are divided into three types, namely, local, regional, and general (global) loads. In scientific literature, one can find detailed characteristics of global loads, including criteria for weights of the loads, their tensity, and intensity. Local loads are often accompanied with a stress on the visual system and a psychemotional strain. It is a well-known fact that the fewer muscles a work involves the sooner fatigue sets in, causing pronounced functional changes in the body. Researches in physiology cite many a fact about harmful influences of long local loads on the supportive-locomotive apparatus and the cardiovascular system [4].

Interrelations between functional changes in the locomotive apparatus and the circulatory system have received the deepest studies for global physical work that involves extensive muscle groups. Based on the results of research conducted at various levels - from the organic level and the body level to the molecular - the researchers [5, 6] formulated theories of adaptive changes occurring in the heart and vessels, depending on various types of physical loads.

In recent years, physical activity patterns in various age groups of population of developed countries have undergone dramatic changes. The researchers [7] have observed an overall reduction in physical activity in all age groups, as well as increased amounts of motions done by small muscle groups (or, local muscle activity, according to generally accepted classification). Present-day labor conditions and living conditions contribute to the role that small muscle groups play amid a sedentary lifestyle in all age groups, with small muscle group constituting up to $1 / 3$ of the overall muscle mass ("local loads"). 


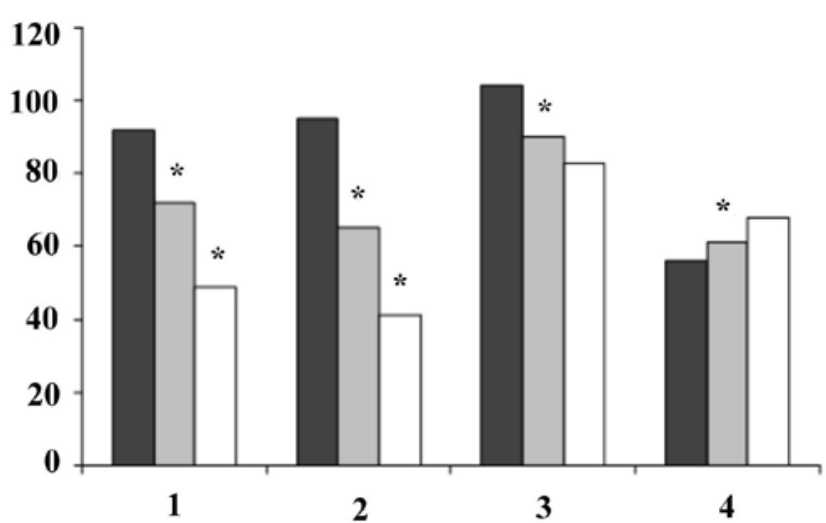

Figure 1. Dynamical work performance against age in male subjects.

Column 1 is for group I, column 2 is for group II, and column 3 is for group III. 1 is the force $\left(\mathrm{kgm}^{*} \mathrm{sec} / 100\right) ; 2$ is the amount of work $(\mathrm{kgm} / 10) ; 3$ is stamina (sec), and 4 is onset of fatigue (sec); * are reliable differences from the previous age group $(\mathrm{P}<0.05)$.

Many a research suggests that local muscle work, especially static muscle work, have a hypertensive effect [8]. A number of researchers have detected an onset of hypertension in those who do jobs that involve long local muscle load. A topical issue, the action of local muscle work on the body depending on age is not being given stuffiest systematic research. The results of that research would shed light on adaptive mechanisms of the blood circulation to local work and subsequent well-justified controlled exercise to be done in the motion mode by all age groups.

It was the aim of this research to study adaptive reactions to local muscle work done by means of a Mosso's ergograph until fatigue set in.

\section{Material and Methods}

We studied reactions of the boold circulatory systems and the central nervous systems to local muscle work of 108 subjects aged between 18 and 74 years. We examined three age groups of adults: group I, aged betwen 18 and 20 years; group II, aged between 30 and 35 years; and group III aged between 60 and 74 years. Prior to the tests, we had obtained informed written consent from all of the subjects.

The subjects were to do a local load test by lifting a load of $1 / 3$ of the "average" weight, their heartbeat rates being 60 to $70 \mathrm{bpm}$ until they stated they were unable to continue the test, and a static load test by holding a load of $1 / 3$ of the maximum weight at a prescribed level until fatigue set in (that is, until they made a verbal statement that they were unable to hold the weight anymore). The conditions in question provided average load for all the age groups [9]. Load measurements were taken by means of a Mosso's ergograph.

Dynamical work was assessed in terms of the following values: the amount of work in $\mathrm{kg} / \mathrm{m}$; stamina in sec, defined as a period of time over with a subject was able to do the work before fatigue set in sec; fatigability defined as a difference percentage between the initial and the last heights at which a subject was able to hold the load.

The values taken into account for static loads were as follows: static stamina in sec, defined as a period of time over which a subject was able to hold the load at a prescribed level; fatigue onset time in sec, and the impulse, defined as the weight in $\mathrm{kg}$ multiplied by the period of time over which a subject was able to hold it, in sec.

Heartbeat rate and arterial blood pressure measurements (according to Korotkov) were taken, and electrocardiograms (ECGs) and electroencephalograms (EEGs) were recorded before, during, and troughout a three-minutes recovery period after a test. Single-channel EEGs were recorded from eight cup electrodes connected to ear electrodes and positioned in strict accordance with the 10-20 system. The function tests were as follows: background record, closed eyes test, and eyes opening. The EEG sampling rate was $250 \mathrm{~Hz}$. We selected at least ten two-secondlong artifact-free EEG record epochs per function test for spectral analysis.

Local work and physiological performance were recorded while the subjects were seated, while they were at rest, and while they were doing the load tests; records were usually made three times: at the start, at the onset of fatigue and before the subjects stated they refused to continue the test, and immediately after a test was over and throughout the first three minutes of rest. EEGs were recorded continuously while a subject was doing a test.

The work done by the subjects on Mosso's ergographs and handgrip dynamometers was similar to their daily work, caused no problem to performing tests, and allowed to record physiological function values immediately when muscle contractions occurred.

The result data processing was done by means of Statistica 6.0 suite (StatSoft, the USA) and SPSS suite. We tested the results for compliance with the normal distribution law and found that the main group was largerly a uniform distribution. We calculated the arithmetic mean (M) of the ordered sample, the standard error of the mean $(\mathrm{m})$, and the Student's t-distribution, while accepting the critical importance value for the verification of statistical hypotheses to be 0.05 .

\section{Results}

Our research showed that the highest performance at average local loads was observed in the young subjects group (aged between 18 and 20) and the mature subjects group (aged between 30 and 35), while fatigue development patterns varied from group to group. The young subjects demonstrated the highest performance in terms of the amounts of work they were able to do, while the mature group was the least prone to fatigue. The closer a human to the age when the body performance reaches its top (18 to 30 ), the sooner fatugue sets in and the deeper it is, and the more considerable amounts of work human bodies can do within short time spans, their capability of getting over fatigue increasing. As a human body ages, the amounts of work human bodies can do decreases, it takes longer for fatigue to set in, while the depth of fatigue that depends on the extent of reduction of the functional performance of the locomotive apparatus remains the same as in young people (Figure 1).

The result of the research suggested that the young group had the highest performance, so as in the dynamical work test. E.g., the force impulse, which was an integral value, was much higher in the young group than in the rest of the subjects. The young and the mature were able to hold heavier loads than the middle-aged, while their stamina was lower $(118.3 \pm 5.6$ and $124.5 \pm 6.9 \mathrm{sec}$ respectively) than that of the middle-aged $(142.6 \pm 5.8 \mathrm{sec})$. 
Table 1. Changes in heartbeat rate and arterial blood pressure after dynamic work in all ages groups

\begin{tabular}{|c|c|c|c|c|c|c|}
\hline \multirow[t]{2}{*}{ Parameters } & \multicolumn{2}{|c|}{ Aged between 18 to 20 years } & \multicolumn{2}{|c|}{ Aged between 30 and 35 years } & \multicolumn{2}{|c|}{ Aged between 60 and 74 years } \\
\hline & 1 & 2 & 1 & 2 & 1 & 2 \\
\hline \multirow[t]{2}{*}{ Heartbeat rate, bpm } & $70.3 \pm 3.4$ & $79.5 \pm 2.5^{*}$ & $63.3 \pm 2.5 * *$ & $68.8 \pm 3.8$ & $74.3 \pm 3.7^{* *}$ & $77.5 \pm 3.2$ \\
\hline & $78.6 \pm 4.3$ & $81.9 \pm 3.7$ & $70.4 \pm 3.0$ & $72.1 \pm 2.7$ & $81.3 \pm 2.9$ & $85.6 \pm 3.5$ \\
\hline \multirow[t]{2}{*}{ Systolic blood pressure, $\mathrm{mmHg}$} & $120.7 \pm 5.2 * *$ & $124.3 \pm 4.7$ & $121.7 \pm 4.1$ & $125.0 \pm 3.5$ & $124.0 \pm 7.2$ & $135.0 \pm 6.8$ \\
\hline & $108.0 \pm 5.3$ & $113.0 \pm 6.7$ & $106.9 \pm 3.8$ & $113.9 \pm 5.2$ & $135.0 \pm 5.4^{* *}$ & $145.0 \pm 6.3$ \\
\hline \multirow[t]{2}{*}{ Diastolic blood pressure, $\mathrm{mmHg}$} & $80.7 \pm 2.9 * *$ & $82.1 \pm 3.4$ & $75.0 \pm 3.4$ & $75.0 \pm 4.2$ & $75.4 \pm 4.8$ & $80.2 \pm 3.9$ \\
\hline & $71.0 \pm 2.5 * *$ & $78.0 \pm 2.3^{*}$ & $76.9 \pm 4.8$ & $78.8 \pm 4.2$ & $87.4 \pm 4.5^{* *}$ & $92.5 \pm 4.1$ \\
\hline \multirow[t]{2}{*}{ Double factor, c.u. } & $85.0 \pm 3.1$ & $98.0 \pm 3.9 *$ & $76.0 \pm 2.5^{* *}$ & $86.0 \pm 3.4^{*}$ & $92.0 \pm 3.5$ & $104.0 \pm 4.8$ \\
\hline & $82.0 \pm 2.8$ & $93.0 \pm 3.2^{*}$ & $74.0 \pm 1.7^{* *}$ & $82.0 \pm 2.8^{*}$ & $109.0 \pm 4.1^{* *}$ & $120.0 \pm 5.2$ \\
\hline
\end{tabular}

1 is before, and 2 - after work; row one is the male subjects, row two is the female subjects. ${ }^{*}-$ are differences between 1 and 2 observed; ${ }^{* *}-$ are differences between age groups observed $(\mathrm{P}<0.05)$. bpm, beats per minute; $c$.u., conventional unit.

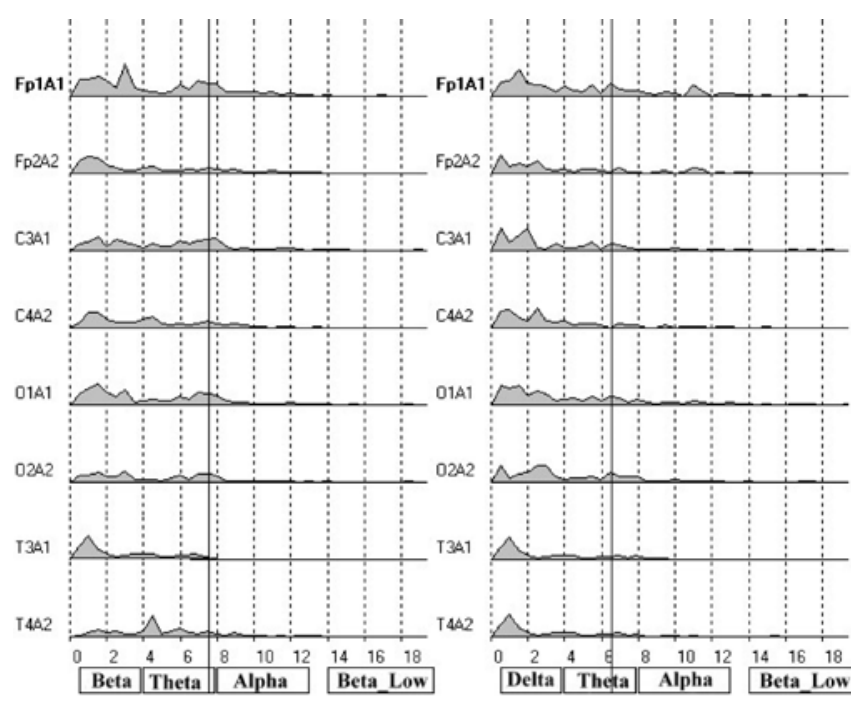

Figure 2. Development of slow-frequency alpha- and theta-waves when doing local loads (patient $A$, age 23 years). $A$ is local load, B is background record, $7.5 \mathrm{~Hz}$ to $6.6 \mathrm{~Hz}, \mathrm{M}: 2$.

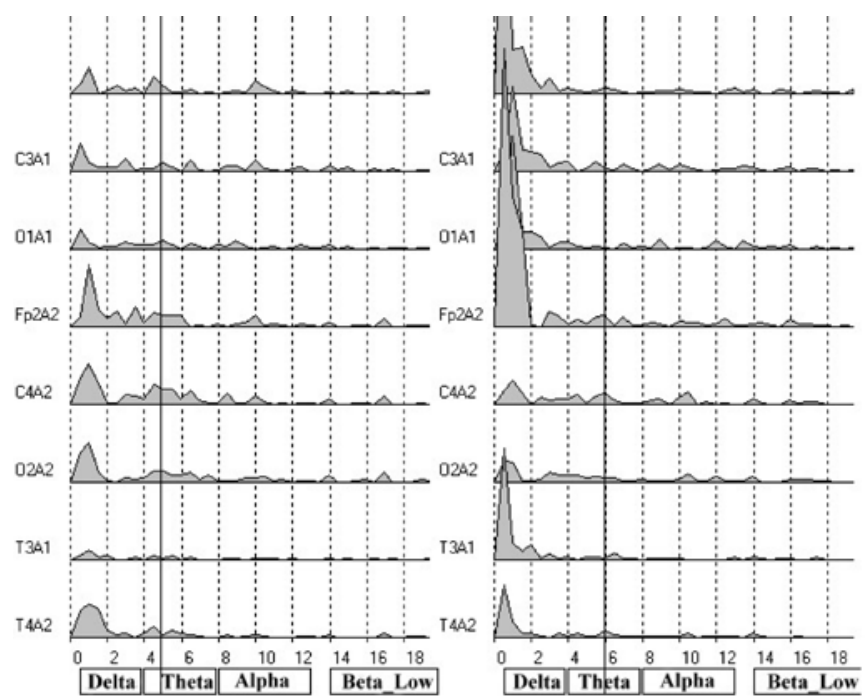

Figure 3. Decreased theta-wave frequency in fronto-central deflections (F2 and C4) at the end of local load (patient B, age 24 years).
Certain heartbeat reactions were observed in all of the subjects. The reactions in question were reliable in males aged between 18 and 20 (Table 1), while heartbeat rates increased less intensely in all of the female age groups than in the male groups. Local dynamical work also triggered arterial blood pressure reactions, especially diastolic ones. Arterial blood pressure reactions were more pronounced in the males with advancing age, with increased blood pressure observed in those aged below 18 to 20 mostly at the onset of fatigue, while in other age groups blood pressure increased upon completion of work as well (Table 1). Rapid recovery was observed in the first minute of rest after a test, while in the second minute, arterial blood pressure values were generally below the initial ones. The lowest blood pressure recovery rate was observed in the subjects aged between 60 and 74. The females above 18 had more pronounced diastolic blood pressure and slower blood pressure recovery rates than in the males. Reliable arterial blood pressure reactions to local work were observed in the middle-aged female subjects.

Reactions to local static work were different in the way that they caused stronger heartbeat rate (beats per minute, BPM) and systolic blood pressure (SBP) reactions than those caused by dynamic work, especially in the young group and the middle-aged group, as well as an increase in the double factor (DF) (DF = BPM $x$ SBP) in almost all of the groups of subjects. These facts indicate that static loads placed higher functional heart stress on most of the subjects than dynamic loads.

Changes in the lengths of ECG intervals observed after local work were most pronounced in $\mathrm{Q}-\mathrm{S}, \mathrm{Q}-\mathrm{T}$, and $\mathrm{R}-\mathrm{R}$ intervals. Static loads, in addition to the above changes, caused shortened diastoles (T-P). Decreased amplitudes of T-waves and increased systolic values were observed in almost all of the subjects after static loads, which indicated that myocardial metabolism occurred under less favorable conditions after static muscle work than after dynamic work.

Our result data suggested that heart function conditions were the most favorable in the mature group of subjects (aged between 30 and 35 ) both at rest and while their muscles received local loads. Functional heart stress, apparently caused by central mechanisms, was observed in the subjects aged between 18 and 20 , and between 60 and 74 .

Spectral frequency analysis of EEGs revealed regular synchronization patterns of alpha-waves. Synchronizations literally formed patterns in specific cortex areas. Periodicity of those patterns varied from subject to subject and ranged from 5 to 70 $\mathrm{sec}$, and 10 to $40 \mathrm{sec}$ in the background mode. Synchronization pattern periods depended on the age and functional states of the 
subjects. The eyes closed tests produced values ranging from 10 to $15 \mathrm{sec}$ on average in all age groups. It is worth noting that the synchronization occurred within the somatosensory area of the cortex, which is the projection area of conducting paths of the thalamic nuclei.

Increased formative periods of synchronization patterns, decreased dominant frequency of alpha-waves, at which synchronization patterns formed, to $8 \mathrm{~Hz}$, and increased lowfrequency activity index of alpha- and theta-waves ( 8.6 to $6.0 \mathrm{~Hz}$ ) were observed on the EEGs in all of the subjects as they were doing local loads.

Periodicity of formation of synchronization patterns had a shared tendency to increase. The alpha-wave index was the highest in prefrontal-central deflections of EEGs, while the increase of the spectral power of alpha-waves reached its highest in the fronto-central deflections.

The analysis of theta-wave activity that occurred when the subject were doing local muscle activities discovered transition phases of the dominant frequency from the alpha- to the thetarange and back again, followed by an increase in the theta-wave. The increase in alpha-wave occurred when most of the subjects had subjective feelings of fatigue onset taking 55 to $70 \%$ on average of the total load test time (Figure 2).

Theta-waves were principally recorded in the fronto-central deflections of the left hemisphere upon completion of a local load. As fatigue set in, a short increase in the electric current frequency was observed in the frontal areas of the cortex, accompanied with depression of theta- and alpha-waves (Figure 3), and the subjects stated that fatigue was clearing up, a sensation similar to "finishing spurt".

The theta-wave index increased by 15 to $20 \%$ of the initial value on average when a subject was doing local load work, with the dominant theta-wave frequency being approx 5 to $6 \mathrm{~Hz}$.

Thus, an increase in the spectral power of slow alpha- and theta-waves was observed as fatigue set in.

A decrease in beta-1-waves was observed in most of the subjects. Dominant slow-frequency beta-waves were observed mostly in the fronto-central deflections of the left hemisphere, with a pronounced activity observed in the frontal areas of both hemispheres at the beta-wave frequency when a subject was doing local muscle work.

It is noteworthy that a pronounced activity was observed in the subjects as they were doing local work in the fronto-central areas of the cortex and synchronization between them at the alpha-wave frequency, combined with that synchronization pattern type in which the fronto-central interaction vector was pronounced.

The common pattern was that the shortening of formative time of synchronization to the initial or even slightly smaller background values was observed in all of the groups during recovery periods after doing local muscle work.

A pronounced increase of the alpha-wave index that predominated in the frontal areas of both hemispheres and an increase of the theta-wave were observed in the recovery period. The activity of the beta-1-wave was the most pronounced in the fronto-central areas of both hemispheres, with a tendency to an increase of the indexes in the central, the frontal, and the occipital deflections of both hemispheres.

\section{Discussion}

The obtained data confirm that there was both age-related and individual difference between adaptive reactions to local work. The chronotropic effect was observed in all of the subjects to a certain extent, namely, within the range of 15 to $25 \mathrm{bpm}$, a change that was a common sign of reactions to local load. Chronotropic reactions were strongest in the young subjects aged between 18 and 20 . Increased arterial blood pressure values were pronounced in the young group and the mature group, while an increase in the DF that was indicative of deteriorated heart function, was observed in all of the age groups as they were doing local work. We proved in our earlier research [10] that long local loads at work contributed to the development of hypertension.

The research results suggested that changes in the heart function occurred during local work due to increased activity of central regulatory influences. Many authors also tend to view fatigue caused by local work in terms of central mechanisms [9, 11]. The research [12] also revealed increased slow-wave activity throughout the cortex as fatigue set in, within theta- and alpha-1and-2-ranges. The researchers provided the data suggesting that global transitions of EEG activity to the range of 4 to $13 \mathrm{~Hz}$ was accompanied with decreased excitation in the cortex and declined cognitive functions [13], which was also observed in subjects who were under the moderate influence of alcohol. The role that serotonin played in the central mechanisms of fatigue was also proved by [14] et al.

Central mechanisms of fatigue involve complex interactions between excitation and inhibition. Slowed down brain waves that are observed at the onset of fatigue are a common sign that cortical inhibition is developing in respective brain areas [11]. The domination of alpha- and theta-waves in frontal areas of both hemispheres while doing local works can be viewed in terms of theory of reversible inhibition in neuronal chains of the cortex.

The researchers [15] link the mechanism of pressor reactions with reflex influences coming from metabolic receptors of active muscles. The researchers [16] see hypertensive reactions to loads as important in prognostication of disease development, while pointing out that enhanced metaboreflex sensitivity was observed in hypertensive humans. It appears that all adaptive body responses are only relatively efficient. Changes in the functional system that receives the heaviest adaptive load, as well as dysfunctions of body organs and systems that are unrelated to muscle work being done, can be "a price to pay" for adaptation to physical loads.

To a higher extent, static effort increases heartbeat rate and blood pressure due to increased afterloads on the heart, while decreasing the stroke volume [17]. The heart responds to an increased afterload by decreasing the contractility and the heartbeat rate, thus improving the cardiac output. Individuals with a low heart rate reserve respond to an increased afterload with a decrease of the impact index and an increase of the final diastolic pressure as they do work. A decreased heart rate reserve may initially appear following a considerable increase of the afterload, even if the static work being done is relatively light.

Central mechanisms of fatigue involve complex interactions between excitation and inhibition. Slowed down brain waves that are observed at the onset of fatigue are a common sign that cortical inhibition is developing in respective brain areas [11]. The domination of alpha- and theta-waves in frontal areas of both 
hemispheres while doing local works can be viewed in terms of theory of reversible inhibition in neuronal chains of the cortex.

The obtained data are indicative of both the predomination of central mechanisms in the formation of adaptive reactions to local muscle work done until fatigue sets in, and of a possibility of a purposeful control over those processes. General physical loads to improve the diastolic function of the heart [18] and the mitochondrial apparatuses of skeletal muscles [2], as well as psychophysical relaxation exercise to normalize the functional state of the central nervous system [19] can be the "means of control"

Facts suggest that physical loads, both general and local, must be planned and administered in order to prevent hypertension. According to our data, the ages between 18 and 20, and between 60 and 74, when the heart receives higher stresses, can be viewed as "critical" age periods. The research [20] has proved that optimal static physical loads develop adaptation that manifests itself as decreased heartbeat rate and blood pressure values while at rest [20]. Physical education and relaxation exercise are recommended to prevent adverse effects of long local loads. Our earlier research [19] contains facts about a beneficial action of psychophysical relaxation exercise on the local workability of muscles.

\section{Conclusion}

The results of our research prove that local muscle work done until fatigues sets in causes pronounced pressor reactions of the circulatory system. Any increased post load on the heart, especially when doing static work, impairs myocardial function. Longer local loads, e.g., received while at work, may result in onset of hypertension. Our data indicate that central nervous mechanisms may underlie adaptive reactions of the circulatory system. Those receiving regular local loads need both blood pressure monitoring and prophylactic campaigns, e.g., physical exercise and relaxation sessions.

\section{Acknowledgments}

The work was supported by Act 211 Government of the Russian Federation, contract № 02.A03.21.0011.

Conflict of interest: none declared.

\section{References}

1. Carrick-Ranson G, Hastings JL, Bhella PS, Fujimoto N, Shibata S, Palmer $\mathrm{MD}$, et al. Impact on lifelong dose of exercise on cardiovascular function during exercise. J Appl Physiol 2014; 116(7): 736-745. https://doi.org/10.1152/japplphysiol.00342.2013.

2. Callahan DM, Bedrin NG, Subramanian M, Berking J, Ades PA, Toth MJ, Miller MS. Age-related structural alterations in human skeletal muscle fibers and mitochondria are sex specific: relationship to single-fiber function. J Appl Physiol 2014; 116(12): 1582-1592. https://doi.org/10.1152/japplphysiol.01362.2013.

3. Baevskii RM, Berseneva AP, Bersenev E Yu, Eshmanova AK. Use of prinsiples of prenozological diagnosis for assessing the functional state of the body under stress conditionc as exemplified by bus drivers. Human Physiology 2009; 35(1): 34-42. https://doi.org/10.1134/S0362119709010071.

4. Chichasova NV. Local treatment of chronic diseases of the musculoskeletal system. Russkij Medicinskij Zhurnal 2001; 9(7-8): 286288. Russian.
5. Medvedev VI. Human adaptation. SPb, Russia: Brain Institute of Russian Academy of Sciences, 2003; 584 p. Russian.

6. Munch GD, Svendsen JH, Damsgaard R, Secher NH, González-Alonso J, Mortensen SP. Maximal heart rate does not limit cardiovascular capacity in healthy humans: insight from right atrial pacing during maximal exercise. J Physiol 2014; 592(2): 377-390. https://doi.org/10.1113/iphysiol.2013.262246.

7. Sherrer J. Physiologic du travail (Ergonomic). Paris: Maisson et Ice Edit, 1967; $527 \mathrm{p}$.

8. Wilborn C, Greenwood M, Wyatt F, Bowden R, Grose D. The effects of exercise intensity and body position on cardiovascular variables during resist resistance exerscise. Journal of Exercise Physiology Online 2004; 7(4): 29-36.

9. Popova TV, Korykalov YI, Kourova OG. Endurance and fatigue caused by local muscular performance in skilled athletes. Human Physiology 2015; 41(6): 680-682. https://doi.org/10.1134/S0362119715060079.

10. Kourova OG, Popova TV, Korykalov YI. Body response to local muscular performance of individuals enagaged in various professional pccupations. Occupational Diseases and Environmental Medicine 2014; 2(3): 71-75. https://doi.org/10.4236/odem.2014.23008.

11. Gorodnichev RM, Petrov DA, Smirnova LV. Investigation of inhibitory processes in the central nervous system with isometric muscle contraction. Vestnik Tverskogo Gosudarstvennogo Unniversiteta 2008; (8): 13-18. Russian. https://elibrary.ru/item.asp?id=11689581.

12. Craig A, Tran $\mathrm{Y}$, Wijesuriya $\mathrm{N}$, Nguyen $\mathrm{H}$. Regional brain wave activity changes associated with fatigue. Psychophysiology 2012; 49(4): 574582. https://doi.org/10.1111/j.1469-8986.2011.01329.x.

13. Dirnberger G, Duregger C, Trettler E, Lindinger G, Lang W. Fatigue in a simple repetitive motor task: A combined electrophysiological and neurophysiological study. Brain Res 2004; 1028(1): 26-30. https://doi.org/10.1016/j.brainres.2004.08.045.

14. Meeusen R, Watson $P$, Hasegawa $H$, Roelands B, Piacentini MF. Central fatigue: the serotonin hypothesis and beyond. Sports Med 2006; 36(10): 881-909. https://www.ncbi.nlm.nih.gov/pubmed/17004850.

15. Choi HM, Stebbins CL, Lee OT, Nho H, Lee JH, Chun JM, Kim KA, Kim JK. Augmentation of the exercise pressor reflex in prehypertension: roles of the muscle metaboreflex and mechanoreflex. Appl Physiol Nutr Metab 2013; 38(2): 209-215. https://doi.org/10.1139/apnm-2012$\underline{0143}$.

16. Sausen MT, Delaney EP, Stillabower ME, Farquhar WB. Enhanced metaboreflex sensitivity in hypertensive humans. Eur J Appl Physiol 2009; 105(3): 351-356. https://doi.org/10.1007/s00421-008-0910-8.

17. Shitya A,Tambovtseva R, Orel V, Gatsunaev A. Central hemodynamic and vascular resistances in the archers in the simulation of the draw. Journal of New Medical Technologies 2015; 22(3): 102-107. Russian. https://doi.org/10.12737/13309.

18. Carrick-Ranson G, Hastings JL, Bhella PS, Fujimoto N, Shibata S, Palmer $\mathrm{MD}$, et al. Impact on lifelong dose of exercise on cardiovascular function during exercise. J Appl Physiol 2014; 116(7): 736-745. https://doi.org/10.1152/japplphysiol.00342.2013.

19. Popova TV, Dovbiy IP, Kourova OG, Koryukalov Yul. Psychophysical training course for students. Theory and Practice of Physical Culture 2016; (1): 43-45. Russian.

20. Jin $Y Z$, Yan $S$, Yuan WX. Effect of isometric handgrip training on resting blood pressure in adults: a meta-analysis of randomized controlled trials. J Sports Med Phys Fitness 2017; 57(1-2): 154-160. https://doi.org/10.23736/S0022-4707.16.05887-4.

\section{Authors:}

Olga G. Kourova - PhD, Associate Professor, Department of Chemistry and Technology, South Ural State University, Chelyabinsk, Russia. http://orcid.org/0000-0002-8544-7631.

Yuri I. Koryukalov - PhD, Doctoral Student, Research Center for Sports Science, Institute of Sport, Tourism, and Service, South Ural State University, Chelyabinsk, Russia. http://orcid.org/0000-0002-4897-2613. 
Tatyana V. Popova - DSc, Professor, South Ural State University, Chelyabinsk, Russia. http://orcid.org/0000-0002-5060-8132. 